CLINICAL HEMORHEOLOGY, VOT. 4, pp. 85-86, 1984

$0271-5198 / 84 \$ 3.00+.00$ Printed in the USA.

Copyright (c) 1984 Pergamon Press Ltd. A11 rights reserved.

\title{
FIFTH INTERNATIONAL CONGRESS OF BIORHEOLOGY
}

SYMPOSIUM ON FHEMORHEOLOGICAL DISORDERS IN CARD COVASCULAR DISEASES

Editor's Epilogue

B. E. Strauer

This symposium has shown that considerable progress in cardiovascular microcirculation has occured during the past years. Nevertheless, from the point of the clinical cardiologist at least three important questions are open and should be tried to be overcome in future:

(1) the analysis of blood rheology

(2) the diagnostics of organ blood flow, and finally

(3) the assessment of therapeutic interventions in rheological diseases as well as the assessment of their clinical significance.

The first point, i.e. the availability of methods appropriate for the measurement of plasma and of blood rheology is most important. The determination of viscosities, of aggregation, of rigidity of blood cells, of deformability and other parameters give the first objective information with regard to altered blood rheology. It is therefore necessary to further focus our interest to these techniques, since they enable the clinician to obtain a measurable correlate for clinically suspected rheological disease.

The second point is the availability of standardized methods for determining regional organ blood flow. These techniques become of special interest, when pharmacological interventions are performed in order to improve or to normalize blood flow by rheologically induced interventions. Particularly, methods for the assessment of the microcirculation of the heart, of the lungs and the peripheral circulation are necessary to be able to realize the normal, the abnormal and therapeutically altered state of organ's microcirculation.

The methods useful and necessary for the quantification of organ perfusion are also necessary and important to answer the third question, that is the evaluation of therapeutical interventions. Give an example: in severe paraproteinemia, the coronary blood flow of the left ventricle may be reduced at rest, however, may be markedly reduced at maximum coronary vasodilatation. This gives insight to be diminished reserve capacity of the coronary vascular bed. The rheological parameters show markedly increased viscosity of both plasma and total blood. Following cytostatics, steroids or plasmapheresis, the coronary blood flow, the coronary reserve as well as the rheological parameters may become considerably improved. 
Consequently the conclusion can be drawn that by normalization of plasma viscosity a normalization of coronary hemodynamics has occured. Studies from our and other clinics have shown that this approach may be relevant for the management of disturbances in coronary and in other organ microcirculation.

However, therapeutical success, as in this case with severe paraproteinemia, must not be realized in a large number of similar diseases, with similar techniques and with similar therapeutical measures. Therefore, for the future, controlled studies are desirable to analyse the influence and importance of abnormal rheological findings on cardiovascular function and, on the other hand, to find out, to what extent rheological abnormalities may occur as a consequence of cardiovascular diseases.

It is our hope that the proceedings of this symposium will provoke the necessary research in this area. 